\title{
Management of Pregnant Women in Covid 19
}

\author{
Nikita Bhokare', Priyanka Naik², Samruddhi Gujar ${ }^{3}$, Komal \\ Meshram ${ }^{4}$ and Roshan Kumar Jha ${ }^{5}$ \\ ${ }^{1}$ Clinical Instructor, DMCON, Nagpur \\ ${ }^{2,3}$ Clinical Instructor, SRMMCON, Sawangi (M) wardha \\ ${ }^{4}$ Depatment of Physiology Datta Meghe Medical College, Nagpur, India \\ ${ }^{5}$ Department of Biochemistry Jawaharlal Nehru Medical College Datta Meghe \\ Institute of Medical Sciences Sawangi (Meghe) Wardha, India \\ Corresponding author email: nikitabhokare530@gmail.com
}

\section{ABSTRACT}

Coronavirus disease 2019 (COVID-19) is a respiratory ailment caused by SARS-CoV-2, a highly contagious and fatal beta coronavirus that has spread fast over the world, necessitating a pandemic proclamation. The aim of this article is to review the most recent research on how COVID 19 impacts pregnant women, as well as the most up-to-date information on treatment options, psychological effects, and the overall impact on healthcare services and resources. These infection prevention and control recommendations are for healthcare facilities that provide obstetric services to pregnant patients with COVID-19 or pregnant Persons Under Investigation (PUI) in obstetric settings like obstetrical triage, labour and delivery, rehabilitation, and inpatient postpartum settings. These concerns are based on the limited evidence available on COVID-19 virus transmission to date, as well as knowledge of other viruses that cause serious respiratory illness, such as influenza, SARS-CoV-2, and the Middle East Respiratory Syndrome coronavirus (MERSCoV).

KEY WORDS: COVID-19, PREGNANT WOMEN, BREAST FEEDING.

\section{INTRODUCTION}

Pregnant women are no more susceptible to illness than the rest of the population. Pregnancy, on the other hand, affects the immune system and the body's response to viral infections in general, which can lead to more severe symptoms in some situations, like with COVID-19. COVID-19 pneumonia in pregnancy has been reported to be milder and to recover quickly. In other forms of coronavirus infection, the hazards to the mother seem to increase during the last trimester of pregnancy (SARS, MERS). Preterm birth has been reported in COVID-19positive women, however it's unclear if the preterm births

Biosc Biotech Res Comm P-ISSN: 0974-6455 E-ISSN: 2321-4007

\section{crossef}

Identifiers and Pagination

Year: 2021 Vol: 14 No (7) Special Issue

Pages: 56-59

This is an open access article under Creative

Commons License Attribn 4.0 Intl (CC-BY). DOI: $h t t p: / / d x . d o i . o r g / 10.21786 / b b r c / 14.7 .13$ were always iatrogenic or if some were unintentional. Women with heart disease who are pregnant are at the highest risk (congenital or acquired).

In June 2020, the Centers for Disease Control and Prevention (CDC) released surveillance data based on pregnancy status on SARS-CoV-2-related outcomes in reproductive-aged women. Among the 326,335 women aged 15 to 44 years who tested positive for SARS-CoV-2, pregnant women were more likely to be hospitalised, admitted to an intensive care unit (ICU), and require mechanical ventilation. However, the overall absolute rise in rates of ICU hospitalisation and mechanical ventilation across pregnant and non-pregnant women was poor (1.5 percent vs. 0.9 percent for ICU admission, respectively, and 0.5 percent vs 0.3 percent for mechanical ventilation, respectively). The death rates from COVID19 were comparable in both pregnant and non-pregnant people. Preterm birth or pregnancy loss were not assessed as pregnancy consequences.
Article Information

Received: $17^{\text {th }}$ April 2021 ccepted after revision: $06^{\text {th }}$ June 2021 
Clinical Staging Of Confirmed Covid- 19 Pregnant Patients

- Mild (URTI)- Groups A, B and C

- Moderate (Pulmonary involvement with hypoxia) - Group D and E

- Severe (Pulmonary involvement with hypoxia with sepsis/septic shock/multi organ dysfunction syndrome) -Group $\mathrm{F}$

\section{MILD - GROUP A}

- Asymtomatic but positive for covid 19

- Isolation ward(ccc)/ Home isolation

- Investigations - CBC, RFT, RBS, LFT, ECG

- Treatment : Tab vitamin C $1000 \mathrm{mg}$ OD, tab zinc 50 mg OD, Vit D 6000 iu stat

- Monitoring of vitals, temp. spo2

\section{MILD - GROUP B}

- Symtomatic / URTI without co morbidity

: Fever, Dry cough, Sore throat, malaise

Headache, Anosmia, loss of taste

Diarrhoea

Red flag signs

1. Neutrophil Lymphocyte ratio $>3.5$

2. Resting tachycardia, 6 min exercise induced hypoxia

3. Spo $2<94 \%$ on room air

- Isolation ward/Home isolation

- Investigations - CBC, RFT, LFT, RBS, ECG, CXR, Spo2 monitoring

\section{Treatment:}

- Tab Favipiravir - no evidence found

- Cap Doxycycline $100 \mathrm{mg}$ bd

- Tab Ivermectin $12 \mathrm{mg}$ Od for 5 days

- Tab vit C, tab zinc, vit D

- Symptomatic treatment as antipyretics, adequate nutrition and appropriate rehydration

- Patient is to be followed up daily for temperature, vitals and spo2.

\section{MILD - GROUP C}

- Symptomatic / URTI with co morbidity

- > 60 years

- Obesity, Diabetes mellitus

- HTN / IHD

- Chronic conditions like CKD, CLD/COPD

- Immunosuppressive drugs and other

- Immunocompromised state

Investigations -

CBC, RFT, RBS, LFT, ECG(daily), CXR ABG, ESR, CRP, LDH, Serum ferritin, D-dimer (If QTc prolongation on ECG , then daily serum electrolytes, ionic calcium and magnesium).

\section{Treatment:}

- Tab Cefixime $200 \mathrm{mg}$ BD OR

- Tab Augmentin 625 mg TDS OR

- Tab Azithromycin 500 mg OD plus
- Tab.Favipiravir (doubtful)

- Tab vit c, tab zinc, vit d

- Inj. LMWH $40 \mathrm{mg}$ SC OD (if D dimer more than 2 to 3 times than normal)

If patient is symptomatic at day 5 , then continue therapy for additional 5 days.

\section{Moderate}

- Pneumonia (LRTI)

- It is associated with presence of dyspnea, fever, cough along with spo $2<94 \%(90-94)$ on room air, $\mathrm{RR}>24 / \mathrm{min}$.

- Investigations - CBC, RFT, RBS, LFT, ECG,(daily) CXR, ABG, ESR, CRP, LDH, Serum ferritin, D-dimer,(4872hrly)

- (If QTc prolongation on ECG, then daily serum electrolytes,

- $\quad$ ionic calcium and magnesium)

- Dedicated covid hospital / SOS ICU

\section{Treatment:}

- Inj Ceftriaxone $1 \mathrm{gm}$ iv BD for 5-10 days +

- Inj Remdesivir ( if benefits outweighs risk)

- $\quad$ vit c, zinc, vit D

- Inj. LMWH 40mg SC OD

- If SPO2 $<94 \%$ consider 1.CARP protocol

- 2.inj.MPS $40 \mathrm{mg}$ iv BD, or Inj dexamethasone $6 \mathrm{mg}$ iv od for 10 days

\section{Severe}

- Severe Pneumonia/ARDS/Septic shock/ Sepsis

It is associated with clinical signs of pneumonia plus RR $>30 /$ min / sever respiratory distresss / Spo2 <90\% on room air.

Red flag signs

1. Neutrophil lymphocyte ratio $>3.5$

2. $\mathrm{PaO} 2 / \mathrm{FiO} 2<300$

3. Raised CRP / serum ferritin / Ddimer / LDH / Triglycerides / Trop I

CPKMB >twice upper limit of normal

Investigations - CBC, RFT, RBS, LFT, ECG,(daily) CXR PA, ABG, Bl. Culture, ESR, CRP, LDH, Serum ferritin, D-dimerTROP I ,CPKMB (If QTc prolongation on ECG, then daily serum electrolytes, ionic calcium and magnesium,

Isolation in ICU

\section{Treatment:}

- Inj. Meropenem $1 \mathrm{gm}$ iv TDS extended infusion over 3 hours +

- Inj remdesivir (if benefits outweighs risk) +

- Inj.LMWH $40 \mathrm{mg}$ s/c od If D dimer is raised three fold then LMWH given in therapeutic dose $40 \mathrm{mg}$ SC BD.

1. CARP protocol

2. inj.MPS /inj dexa

If raised IL6, d dimers and ferritin, then add inj Toclizumab 9if benefit outweighs risk) (400 mg in 100 
ml NS over 1 hour)

- $\quad$ Vit c, zinc, Vit d

\section{Management}

\section{Antenatal care}

- Women should be advised to attend frequent antenatal treatment, customized to a minimum, at the discretion of the maternal care provider at 12 , 20,28 , and 36 weeks of pregnancy unless they meet existing self-isolation standards.

- Unless symptoms (other than a persistent cough) become serious, appointments for women who have had symptoms should be postponed until 7 days after the commencement of symptoms. It's crucial to keep track of how many times the foetus kicks.

- If she has to go to the hospital, she can either drive herself or call 108, telling the ambulance crew of her condition.

- Appointments for women who have self-quarantined because a family member may have COVID-19 symptoms should be postponed for 14 days.

- Any woman who has had her normal appointment pushed out for more than three weeks should be notified. (In rural regions, ANMs/ASHAs may call people or make regular visits with PPE to their houses.)

- Even if a woman has previously tested negative for COVID-19, if she develops symptoms again, COVID19 should be considered.)Following the resolution of acute illness, referral to antenatal ultrasound services for foetal development surveillance is recommended after 14 days. 6,7

\section{Intrapartum Care}

- A multidisciplinary team, including an infectious diseases or medical professional, should assess the severity of COVID-19 symptoms.

- $\quad$ The delivery should preferably take place at a tertiary care facility.

- $\quad$ The mother's temperature, respiration rate, and oxygen saturations are all monitored. Cardiotocographbased electronic foetal monitoring (CTG)

- Maintain an oxygen saturation of $>94 \%$ by adjusting oxygen therapy as required.

- If the woman exhibits sepsis symptoms, examine and treat her as directed in the sepsis in pregnancy recommendations, but additionally consider active COVID-19 as a possible cause of sepsis and investigate accordingly.

- In the presence of coronaviruses, there is no indication that epidural or spinal analgesia or anaesthesia is contraindicated. In women with suspected or confirmed COVID19, epidural analgesia should be prescribed during labour to reduce the need for general anaesthesia if an urgent delivery is required.

- If a caesarean section or other surgical treatment is necessary, the surgery should be carried out while wearing personal protective equipment (PPE).

- If a symptomatic woman is growing exhausted or hypoxic, an individualized decision on elective assisted birth to decrease the duration of the second stage of labor should be made.

Postnatal Management: It's unknown whether COVID19-positive newborns are more likely to develop significant problems. Because of the contact with contagious respiratory secretions, transmission after delivery is a problem. Facilities should consider temporarily separating the mother who has reported COVID-19 or is a PUI from her daughter (e.g., separate rooms) until the mother's transmission-based measures are removed.9

\section{Precautions for Pregnant Women}

- Using an alcohol-based hand rub or soap and water to wash your hands regularly.

- $\quad$ Maintaining a safe distance between yourself and others when avoiding crowded areas. When keeping enough physical space between yourself and others is impossible, wear a non-medical cloth mask. Do not touch your eyes, nose, or mouth.

- Taking care of one's lungs. When you cough or sneeze, cover your mouth and nose with your bent elbow or a tissue. Then throw away the used tissue right away.

- If you have a fever, a cough, or are having trouble breathing, see a doctor as soon as possible. Before visiting a health centre, call ahead and follow the local health authority's instructions.

- Pregnant women and women who have recently given birth should keep their regular doctor's appointments, according to local laws, and take appropriate precautions to prevent the virus from spreading.

\section{Vaccination in Pregnancy and Lactation}

- Pregnant women have been excluded from trials evaluating COVID-19 vaccines, thus safety and efficacy data are limited.

- Up to date suggest COVID-19 vaccination for pregnant women rather than deferring vaccination until after delivery, particularly for those at higher risk of exposure or severe disease if infected.

- Although pregnancy itself is associated with an increased risk of severe infection, some patients may reasonably elect to defer vaccination after weighing their personal risk of COVID-19 exposure and disease severity against the limited data regarding the safety and efficacy of COVID-19 vaccines during pregnancy.

- Vaccination should be timed so that patients do not receive COVID-19 vaccines within 14 days of receipt of a routinely administered vaccine.

- However, a shorter interval between COVID-19 vaccines and other vaccines is reasonable when timely administration of another vaccine is important (eg, tetanus vaccination during wound management) or if it would avoid unnecessary delays in COVID-19 vaccination.

- Vaccination is not thought to affect fertility, and it is not necessary to delay pregnancy after vaccination. 


\section{Recommendation:}

The World Health Organization and the US Centres for Disease Control and Prevention encourage pregnant women to follow the same infection-prevention guidelines as the general public, such as covering coughs, avoiding contact with sick people, and washing hands with soap and water or sanitizer.

\section{CONCLUSION}

Infection with COVID-19 during pregnancy increases the risk of pregnancy complications such as preterm birth, PPROM, and, in extreme circumstances, maternal death. There is no proof that SARS-CoV-2 infection can be transmitted vertically to an unborn child.

\section{REFERENCES}

Centers for Disease Control and Prevention. Care for breastfeeding women: interim guidance on breastfeeding and breast milk feeds in the context of COVID-19. 2020. Available at: https://www.cdc.gov/coronavirus/2019ncov/hcp/care-for-breastfeeding-women.html. Accessed August 26, 2020.

Centers for Disease Control and Prevention. Evaluation and management considerations for neonates at risk for COVID-19. 2020. https://www.cdc.gov/ coronavirus/2019-ncov/hcp/caring-for-newborns.html. Accessed August 26, 2020.

Centres for Disease Control and Prevention. Interim Considerations for Infection Prevention and Control of Coronavirus Disease 2019 (COVID-19) in Inpatient Obstetric Healthcare Settings. [Online] April 4, 2020.
Accessed on April 5, 2020.

Chawla D, et al. Perinatal-Neonatal Management of COVID-19 Infection. Indian Paediatrics 2020 pii: S097475591600154. [Online] April 1, 2020. Accessed on April 5, 2020.

Favre G, Pomar L, Qi X, Nielsen-Saines K, Musso D, Baud D. Guidelines for pregnant women with suspected SARS-CoV-2 infection. Lancet Infectious Diseases 2020 pii:S1473-3099(20)30157-2. [Online] March 3, 2020. Accessed on April 5, 2020.

Government of India, Ministry of Health \& Family Welfare, Directorate General of Health Services (EMR Division). Revised Guidelines on Clinical Management ofCOVID-19. [Online] March 31, 2020. Accessed on April 5, 2020

https://obgyn.onlinelibrary.wiley.com/doi/full/10.1111/ jog. 14321

https://rebelem.com/covid-19-clinical-therapeuticstaging-proposal-and-treatment/

https://www.ncbi.nlm.nih.gov/pmc/articles/ PMC7842457/

https://www.uptodate.com/contents/covid-19-andpregnancy-questions-and-answers\#H1091745192

The Federation of Obstetric and Gynaecological Societies of India. Good Clinical Practice recommendation on Pregnancy with COVID-19 Infection; Version 1; [Online] March 28, 2020. Accessed on April 5, 2020.

The Royal College of Midwives, Royal College of Obstetrics and Gynaecology, Royal College of Paediatrics and Child Health, Royal College of Anaesthetists. Coronavirus COVID-19 Infection in Pregnancy; Version 5. [Online] March 28, 2020. Accessed on April 5, 2020. 